PROCEEDINGS OF THE

AMERICAN MATHEMATICAL SOCIETY

Volume 140, Number 8, August 2012, Pages 2671-2686

S 0002-9939(2011)11129-8

Article electronically published on December 22, 2011

\title{
STRONGLY CENTRAL SETS AND SETS OF POLYNOMIAL RETURNS MOD 1
}

\author{
VITALY BERGELSON, NEIL HINDMAN, AND DONA STRAUSS
}

(Communicated by Jim Haglund)

\begin{abstract}
Central sets in $\mathbb{N}$ were introduced by Furstenberg and are known to have substantial combinatorial structure. For example, any central set contains arbitrarily long arithmetic progressions, all finite sums of distinct terms of an infinite sequence, and solutions to all partition regular systems of homogeneous linear equations. We introduce here the notions of strongly central and very strongly central, which as the names suggest are strictly stronger than the notion of central. They are also strictly stronger than syndetic, which in the case of $\mathbb{N}$ means that gaps are bounded.

Given $x \in \mathbb{R}$, let $w(x)=x-\left\lfloor x+\frac{1}{2}\right\rfloor$. Kronecker's Theorem says that if $1, \alpha_{1}, \alpha_{2}, \ldots, \alpha_{v}$ are linearly independent over $\mathbb{Q}$ and $U$ is a nonempty open subset of $\left(-\frac{1}{2}, \frac{1}{2}\right)^{v}$, then $\left\{x \in \mathbb{N}:\left(w\left(\alpha_{1} x\right), \ldots, w\left(\alpha_{v} x\right)\right) \in U\right\}$ is nonempty, and Weyl showed that this set has positive density. We show here that if $\overline{0}$ is in the closure of $U$, then this set is strongly central. More generally, let $P_{1}, P_{2}, \ldots, P_{v}$ be real polynomials with zero constant term. We show that

$$
\left\{x \in \mathbb{N}:\left(w\left(P_{1}(x)\right), \ldots, w\left(P_{v}(x)\right)\right) \in U\right\}
$$

is nonempty for every open $U$ with $\overline{0} \in c \ell U$ if and only if it is very strongly central for every such $U$ and we show that these conclusions hold if and only if any nontrivial rational linear combination of $P_{1}, P_{2}, \ldots, P_{v}$ has at least one irrational coefficient.
\end{abstract}

\section{INTRODUCTION}

In 1884 Kronecker [16] proved the theorem stated in the abstract. It is usually stated in terms of the fractional part of a real number $x$, that is $x-\lfloor x\rfloor$. We have moved the resulting interval down from $[0,1)$ to $\left[-\frac{1}{2}, \frac{1}{2}\right.$ ) (and deal with the function $\left.w(x)=x-\left\lfloor x+\frac{1}{2}\right\rfloor\right)$ because we are concerned with sets that are close to zero in the circle group $\mathbb{T}=\mathbb{R} / \mathbb{Z}$ and it is more convenient to talk about the interval $(-\epsilon, \epsilon)$ than the corresponding $[0, \epsilon) \cup(1-\epsilon, 1) \subseteq[0,1)$. One can show that if $1, \alpha_{1}, \alpha_{2}, \ldots, \alpha_{v}$ are linearly independent over $\mathbb{Q}$ and $U$ is a nonempty open subset of $\left(-\frac{1}{2}, \frac{1}{2}\right)^{v}$, then $\left\{x \in \mathbb{N}:\left(w\left(\alpha_{1} x\right), \ldots, w\left(\alpha_{v} x\right)\right) \in U\right\}$ has bounded gaps, and in fact is an $\mathrm{IP}_{+}^{*}$ set (see [3, Theorem 3.12]). A set $A \subseteq \mathbb{N}$ is an IP set if and only if there exists a sequence $\left\langle x_{n}\right\rangle_{n=1}^{\infty}$ in $\mathbb{N}$ such that $F S\left(\left\langle x_{n}\right\rangle_{n=1}^{\infty}\right) \subseteq A$ where $F S\left(\left\langle x_{n}\right\rangle_{n=1}^{\infty}\right)=\left\{\sum_{n \in F} x_{n}: F \in \mathcal{P}_{f}(\mathbb{N})\right\}$, and for any set $X, \mathcal{P}_{f}(X)$ is the set of finite nonempty subsets of $X$. The same definition applies to an arbitrary semigroup $(S,+)$. (We denote the operation by + because we shall be mostly concerned with

Received by the editors June 17, 2010 and, in revised form, March 16, 2011.

2010 Mathematics Subject Classification. Primary 05D10.

The first two authors acknowledge support received from the National Science Foundation via Grants DMS-0901106 and DMS-0852512 respectively. 
subsemigroups of $\mathbb{R}$ under addition, but we do not assume that $S$ is commutative. In the expression $\sum_{n \in F} x_{n}$, the sum is taken in increasing order of indices.) A set is an $I P^{*}$ set if and only if it meets every IP set, equivalently, its complement is not an IP set. And a subset $A$ of $S$ is an IP* set if and only if it is an IP* set or there is some $x \in S$ such that $-x+A$ is an $\mathrm{IP}^{*}$ set, where $-x+A=\{y \in S: x+y \in A\}$. (In spite of the + subscript, $\mathrm{IP}_{+}^{*}$ is a weaker property than $\mathrm{IP}^{*}$.)

A subset $A$ of $\mathbb{N}$ is thick if and only if it contains arbitrarily long blocks of integers and is syndetic if an only if there is a bound on the gaps of $A$. More generally, a subset $A$ of a discrete semigroup $(S,+)$ is thick if and only if for every finite subset $F$ of $S$, there is some $x \in S$ such that $F+x \subseteq A$. And $A$ is syndetic if and only if there is a finite subset $F$ of $S$ for which $S=\bigcup_{t \in F}(-t+A)$. Thus, $A$ is syndetic if and only if $S \backslash A$ is not thick. It is easy to see that any thick set is an IP set and consequently that any $\mathrm{IP}^{*}$ set is syndetic.

For subsets of a commutative semigroup, the properties of being thick and syndetic are both upward and downward translation invariant, that is if $A$ has one of these properties and $t \in S$, then $t+A$ and $-t+A$ also have that same property. The properties IP and IP* are not translation invariant. (The even integers are IP* while the odd integers do not contain any $\{x, y, x+y\}$.) Notice that, as a consequence of the above discussion, any $\mathrm{IP}_{+}^{*}$ set is syndetic. (In fact, by 3 , Theorem 2.20 ], the property of being an $\mathrm{IP}_{+}^{*}$ set in $\mathbb{N}$ is strictly stronger than being syndetic.) What was actually established in [3, Theorem 3.12] is that the set in question is not just an $\mathrm{IP}_{+}^{*}$ set, but has a much stronger property, namely that all downward translates by members of the given set yield an $\mathrm{IP}^{*}$ set.

Theorem 1.1. Let $v \in \mathbb{N}$ and assume that $1, \alpha_{1}, \alpha_{2}, \ldots, \alpha_{v}$ are linearly independent over $\mathbb{Q}$. Let $U$ be an open subset of $\left(-\frac{1}{2}, \frac{1}{2}\right)^{v}$, and let

$$
A=\left\{x \in \mathbb{N}:\left(w\left(\alpha_{1} x\right), w\left(\alpha_{2} x\right), \ldots, w\left(\alpha_{v} x\right)\right) \in U\right\} .
$$

Then for every $x \in A,-x+A$ is an $I P^{*}$ set.

The following two polynomial variations on the theme of Kronecker have a direct relation to the main result of this paper.

Theorem 1.2 (See [3, Theorem 3.15]). Let $v \in \mathbb{N}$, let $P_{1}, P_{2}, \ldots, P_{v}$ be real polynomials, and let $U$ be a nonempty open subset of $\left(-\frac{1}{2}, \frac{1}{2}\right)^{v}$. Assume that any nontrivial linear combination of $\left\{P_{u}: u \in\{1,2, \ldots, v\}\right\}$ over $\mathbb{Q}$ has at least one irrational coefficient. Then $\left\{x \in \mathbb{N}:\left(w\left(P_{1}(x)\right), w\left(P_{2}(x)\right), \ldots, w\left(P_{v}(x)\right)\right) \in U\right\}$ is an IP* set.

Theorem 1.3. Let $v \in \mathbb{N}$, and let $P_{1}, P_{2}, \ldots, P_{v}$ be real polynomials with zero constant term. Let $U$ be a neighborhood of $\overline{0}$ in $\left(-\frac{1}{2}, \frac{1}{2}\right)^{v}$, and let

$$
A=\left\{x \in \mathbb{N}:\left(w\left(P_{1}(x)\right), w\left(P_{2}(x)\right), \ldots, w\left(P_{v}(x)\right)\right) \in U\right\} .
$$

Then $A$ is an $I P^{*}$ set.

Proof [12, Theorem 2.19]. (This is also a special case of [7, Theorem D].)

In this paper we deal with an assumption about $U$ which is midway between the assumptions of Theorems 1.2 and 1.3. that is, we shall assume that $\overline{0}$ is in the closure of $U$. We cannot, of course, hope to get a conclusion that $A$ is an IP* set since there are many disjoint open sets $U$ with $\overline{0} \in c \ell U$. (It is easy to see, using the 
algebraic characterization of $\mathrm{IP}^{*}$ which we will present below, that the intersection of two IP* sets is an IP* set.) What we do obtain is that, with the same assumption on the polynomials as in Theorem 1.2, $A$ is very strongly central. (And we show that that assumption is necessary.)

To describe the central, strongly central, and very strongly central properties, we need to pause and briefly introduce the algebraic structure of the Stone-Cech compactification $\beta S$ of a discrete semigroup $(S,+)$. Given any discrete semigroup $S$ we take $\beta S$ to be the set of ultrafilters on $S$, identifying the points of $S$ with the principal ultrafilters. The topology on $\beta S$ has a basis consisting of $\{c \ell A: A \subseteq S\}$, where $c \ell A=\{p \in \beta S: A \in p\}$. The operation on $S$ extends to $\beta S$, making $\beta S$ a right topological semigroup with $S$ contained in its topological center. That is, for each $p \in \beta S$, the function $\rho_{p}: \beta S \rightarrow \beta S$ is continuous where for $q \in \beta S$, $\rho_{p}(q)=q+p$. And, for each $x \in S$, the function $\lambda_{x}: \beta S \rightarrow \beta S$ is continuous where for $q \in \beta S, \lambda_{x}(q)=x+q$. (In spite of the fact that we are denoting the extension by + , the operation on $\beta S$ is very unlikely to be commutative. The center of $(\beta \mathbb{N},+)$ is $\mathbb{N}$.) Given $p$ and $q$ in $\beta S$ and $A \subseteq S, A \in p+q$ if and only if $\{x \in S:-x+A \in q\} \in p$. See [2], 3], [4], or [15] for an introduction to the algebraic structure of $\beta S$ and for unfamiliar facts about the structure of $\beta S$ encountered here, with the caution that the first three references take $\beta S$ to be left topological rather than right topological.

Any compact right topological semigroup has idempotents [11, Lemma 1]. A set $A \subseteq S$ is an IP set if and only if $A$ is a member of an idempotent in $\beta S$. (For the equivalence with the elementary definition given earlier, see [15, Theorem 5.12].) Consequently, $A$ is an $\mathrm{IP}^{*}$ set if and only if it is a member of every idempotent in $\beta S$.

Central subsets of $\mathbb{N}$ were introduced by Furstenberg in [12] and were defined in terms of notions of topological dynamics. In [5] an equivalent characterization in terms of the algebra of $\beta \mathbb{N}$ was established (with the assistance of $\mathrm{B}$. Weiss). We take that characterization to be the definition. In order to present it, we need to describe a little more about the algebra of $\beta S$. Any compact right topological semigroup $T$ has a smallest two sided ideal $K(T)$ which is the union of all of the minimal left ideals and is also the union of all of the minimal right ideals. The intersection of any minimal left ideal with any minimal right ideal is a group. In particular, there are idempotents in $K(T)$. Such idempotents are called minimal. A subset $L$ of $T$ is a minimal left ideal if and only if $L=T+p$ for some minimal idempotent $p$.

Definition 1.4. Let $S$ be a discrete semigroup. A set $A \subseteq S$ is central if and only if $A$ is a member of some minimal idempotent.

From the definition it is immediate that if $\mathbb{N}$ is divided into finitely many sets, at least one of these is central. Central subsets of $\mathbb{N}$ have substantial combinatorial properties, which are consequences of the original Central Sets Theorem 12, Proposition 8.21]. (A stronger version can be found in [10.) For example, as mentioned in the abstract, given any partition regular system of homogeneous linear equations with integer coefficients, a solution can be found in any central set. In fact, there are countably many such systems. If they are enumerated, one may find a solution set for each system with the property that any sum of finitely many terms chosen from distinct solution sets lie in a given central set. See Chapters 14 through 16 of 15] for more examples of the structure that can be found in any central set. 
A subset $A$ of $\mathbb{N}$ is piecewise syndetic if and only if there exists $k \in \mathbb{N}$ such that $\bigcup_{t=1}^{k}(-t+A)$ is thick. These sets are interesting because they are precisely the sets whose closure meets $K(\beta \mathbb{N})$. It is a consequence of [15. Theorem 4.43] that $A$ is piecewise syndetic if and only if it is a translate of a central subset of $\mathbb{N}$.

In Section 2 we will introduce the notions of strongly central and very strongly central subsets of $S$ and show that these notions satisfy the implications suggested by their names.

Our main result is the following theorem, which we will prove in Section 3.

Theorem 3.2. Let $v \in \mathbb{N}$ and for $u \in\{1,2, \ldots, v\}$, let $P_{u}$ be a polynomial with real coefficients and zero constant term. The following statements are equivalent.

(a) Whenever $U$ is an open subset of $\mathbb{T}^{v}$ with $\overline{0} \in c \ell U$, $\left\{x \in \mathbb{N}:\left(w\left(P_{1}(x)\right), w\left(P_{2}(x)\right), \ldots, w\left(P_{v}(x)\right)\right) \in U\right\}$ is very strongly central.

(b) Whenever $U$ is an open subset of $\mathbb{T}^{v}$ with $\overline{0} \in c \ell U$, $\left\{x \in \mathbb{N}:\left(w\left(P_{1}(x)\right), w\left(P_{2}(x)\right), \ldots, w\left(P_{v}(x)\right)\right) \in U\right\}$ is strongly central.

(c) Whenever $U$ is an open subset of $\mathbb{T}^{v}$ with $\overline{0} \in \mathrm{clU}$, $\left\{x \in \mathbb{N}:\left(w\left(P_{1}(x)\right), w\left(P_{2}(x)\right), \ldots, w\left(P_{v}(x)\right)\right) \in U\right\}$ is central.

(d) Whenever $U$ is an open subset of $\mathbb{T}^{v}$ with $\overline{0} \in \mathrm{clU}$, $\left\{x \in \mathbb{N}:\left(w\left(P_{1}(x)\right), w\left(P_{2}(x)\right), \ldots, w\left(P_{v}(x)\right)\right) \in U\right\} \neq \emptyset$.

(e) Any nontrivial linear combination of $\left\{P_{u}: u \in\{1,2, \ldots, v\}\right\}$ over $\mathbb{Q}$ has at least one irrational coefficient.

\section{Strongly AND VERY STRONGLY CENTRAL SETS}

Most of the results in this section are valid for an arbitrary semigroup. We shall denote the semigroup operation by + because we wish to apply the results of this section to $(\mathbb{N},+)$. However, we are not assuming that $S$ is commutative.

Definition 2.1. Let $S$ be a discrete semigroup. A set $A \subseteq S$ is strongly central if and only if for every minimal left ideal $L$ of $\beta S$ there is an idempotent in $c \ell A \cap L$; that is, there is an idempotent $p \in L$ such that $A \in p$.

Since a set is central in $\mathbb{N}$ if and only if it is a member of an idempotent in some minimal left ideal $L$ of $\beta \mathbb{N}$, and since there are $2^{\mathfrak{c}}$ minimal left ideals of $\beta \mathbb{N}[8$, the assertion that a set is a member of an idempotent in every minimal left ideal $L$ of $\beta \mathbb{N}$ is considerably stronger than the assertion that it is central. To see this, let $p$ be any idempotent in $K(\beta \mathbb{N})$, and let $L$ be a minimal left ideal of $\beta \mathbb{N}$ for which $p \notin L$. Since $L$ is closed, by [15, Corollary 2.6], there is a subset $A$ of $\mathbb{N}$ for which $A \in p$ and $L \cap c \ell A=\emptyset$. So $A$ is central but not strongly central.

We have already noted that any $\mathrm{IP}_{+}^{*}$ set is syndetic. It is also true that every strongly central set is syndetic. (By 6, Theorem 2.9], $A$ is syndetic if and only if for every left ideal $L$ of $\beta S, c \ell A \cap L \neq \emptyset$, and thus $A$ is thick if and only if $c \ell A$ contains a left ideal of $\beta S$.)

Theorem 2.2. Let $(S,+)$ be a semigroup, let $A$ be a strongly central subset of $S$, and let $B$ be a thick subset of $S$. Then $A \cap B$ is central.

Proof. By [6, Theorem 2.9(c)], there is a minimal left ideal $L$ of $\beta S$ such that $L \subseteq c \ell B$. Pick an idempotent $p \in L \cap c \ell A$. 
Question 2.3. Let $(S,+)$ be a semigroup and let $A \subseteq S$. If $A \cap B$ is central for every thick set $B$, must $A$ be strongly central?

As we have mentioned, central sets were originally defined by Furstenberg in terms of notions from topological dynamics. Specifically, the definition in [12] (for the semigroup $(\mathbb{N},+)$ ), is condition (b) of Theorem 2.5, except that in [12] the space $X$ was assumed to be a metric space.

\section{Definition 2.4.}

(1) A dynamical system is a pair $\left(X,\left\langle T_{s}\right\rangle_{s \in S}\right)$ such that

(a) $X$ is a compact Hausdorff space;

(b) $S$ is a semigroup;

(c) for each $s \in S, T_{s}$ is a continuous function from $X$ to $X$; and

(d) for all $s, t \in S, T_{s} \circ T_{t}=T_{s+t}$.

(2) If $\left(X,\left\langle T_{s}\right\rangle_{s \in S}\right)$ is a dynamical system, a point $y \in X$ is uniformly recurrent if and only if for every neighborhood $U$ of $y,\left\{s \in S: T_{s}(y) \in U\right\}$ is syndetic.

(3) If $\left(X,\left\langle T_{s}\right\rangle_{s \in S}\right)$ is a dynamical system, points $x$ and $y$ of $x$ are proximal if and only if there is a net $\left\langle s_{\iota}\right\rangle_{\iota \in I}$ in $S$ such that the nets $\left\langle T_{s_{\iota}}(x)\right\rangle_{\iota \in I}$ and $\left\langle T_{S_{\iota}}(y)\right\rangle_{\iota \in I}$ converge to the same point of $X$.

(4) A dynamical system $\left(X,\left\langle T_{s}\right\rangle_{s \in S}\right)$ is minimal if and only if there does not exist a nonempty proper closed subset $Y$ of $X$ such that $T_{s}[Y] \subseteq Y$ for all $s \in S$.

(5) If $X$ is a compact Hausdorff space and $T$ is a continuous function from $X$ to $X$, then $T$ is said to be a minimal transformation if and only if $\left(X,\left\langle T^{n}\right\rangle_{n \in \mathbb{N}}\right)$ is a minimal dynamical system.

As we mentioned in the introduction, the special case of the following theorem, which has $S=\mathbb{N}$, was established in $[5]$.

Theorem 2.5. Let $(S,+)$ be a semigroup and let $A \subseteq S$. The following statements are equivalent.

(a) A is central.

(b) There exist a dynamical system, $\left(X,\left\langle T_{s}\right\rangle_{s \in S}\right)$, points $x$ and $y$ in $X$, and a neighborhood $U$ of $y$ in $X$ such that $y$ is uniformly recurrent, $x$ and $y$ are proximal, and $A=\left\{s \in S: T_{s}(x) \in U\right\}$.

Proof [19, Theorem 2.4]. Or see [15, Theorem 19.27].

We have a dynamical characterization of strongly central sets in terms of the following notion introduced by R. McCutcheon in [18].

Definition 2.6. Let $\mathcal{A}$ be a family of subsets of the semigroup $(S,+)$. Then $\mathcal{A}$ is collectionwise left thick if and only if any intersection of finitely many members of $\mathcal{A}$ is thick.

Lemma 2.7. Let $\mathcal{A}$ be a family of subsets of the semigroup $(S,+)$. Then $\mathcal{A}$ is collectionwise left thick if and only if there is a left ideal $L$ of $\beta S$ such that $L \subseteq$ $\bigcap_{A \in \mathcal{A}} c \ell A$.

Proof. The sufficiency is trivial because a subset of $S$ is thick if and only if its closure contains a left ideal of $\beta S$.

For the necessity, let $D=\mathcal{P}_{f}(S) \times \mathcal{P}_{f}(\mathcal{A})$, and direct $D$ by agreeing that $(F, \mathcal{F}) \leq$ $\left(F^{\prime}, \mathcal{F}^{\prime}\right)$ if and only if $F \subseteq F^{\prime}$ and $\mathcal{F} \subseteq \mathcal{F}^{\prime}$. For each $(F, \mathcal{F}) \in D$, we have that 
$\bigcap \mathcal{F}$ is thick, so pick $t_{(F, \mathcal{F})} \in S$ such that $F+t_{(F, \mathcal{F})} \subseteq \bigcap \mathcal{F}$. Let $q$ be a limit point of the net $\left\langle t_{(F, \mathcal{F})}\right\rangle_{(F, \mathcal{F}) \in D}$. We claim that $\beta S+q \subseteq \bigcap_{A \in \mathcal{A}} c \ell A$ for which it suffices that $S+q \subseteq \bigcap_{A \in \mathcal{A}} c \ell A$, because $\beta S+q=c \ell(S+q)$. So let $s \in S$ and $A \in \mathcal{A}$, and suppose that $s+q \notin c \ell A$. Let $B=S \backslash A$. Then $-s+B \in q$, so pick $(F, \mathcal{F}) \in D$ such that $(F, \mathcal{F}) \geq(\{s\},\{A\})$ and $t_{(F, \mathcal{F})} \in(-s+B)$. Then $s \in F$ so $s+t_{(F, \mathcal{F})} \in \bigcap \mathcal{F} \subseteq A$ while $s+t_{(F, \mathcal{F})} \in B$, a contradiction.

Recall that if $p \in \beta S$ and $\left\langle x_{s}\right\rangle_{s \in S}$ is an indexed family in the compact Hausdorff space $X$, then $p$ - $\lim _{s \in S} x_{s}=y$ if and only if for every neighborhood $U$ of $y$, $\left\{s \in S: x_{s} \in U\right\} \in p$.

Theorem 2.8. Let $(S,+)$ be a semigroup, and let $B \subseteq S$. Then $B$ is strongly central if and only if whenever $\mathcal{A}$ is a collectionwise left thick family of subsets of $S$ and $\left(X,\left\langle T_{s}\right\rangle_{s \in S}\right)$ is a dynamical system, there exists a point $y \in X$ such that for each $A \in \mathcal{A}$ and each neighborhood $U$ of $y,\left\{s \in A \cap B: T_{s}(y) \in U\right\} \neq \emptyset$.

Proof. Necessity. Let $\mathcal{A}$ and $\left(X,\left\langle T_{s}\right\rangle_{s \in S}\right)$ be given. By Lemma 2.7 pick a minimal left ideal $L$ of $\beta S$ such that $L \subseteq \bigcap_{A \in \mathcal{A}} c \ell A$ and pick an idempotent $p \in L \cap$ $c \ell B$. Pick any $x \in X$ and let $y=p$ - $\lim _{s \in S} T_{s}(x)$. Then by [15, Theorem 19.11], $p-\lim _{s \in S} T_{s}(y)=y$. Let $U$ be a neighborhood of $y$. Then $A \in p, B \in p$, and $\left\{s \in S: T_{s}(y) \in U\right\} \in p$, so the intersection of these three sets is nonempty.

Sufficiency. Let $L$ be a minimal left ideal of $\beta S$. We shall show that there is an idempotent $p \in L \cap c \ell B$. Let $\mathcal{A}=\{A \subseteq S: L \subseteq c \ell A\}$. By Lemma 2.7, $\mathcal{A}$ is collectionwise left thick. By [15, Theorem 19.8], $\left(L,\left\langle\lambda_{s}^{\prime}\right\rangle_{s \in S}\right)$ is a dynamical system, where $\lambda_{s}^{\prime}$ is the restriction of $\lambda_{s}$ to $L$. Pick a point $y \in L$ such that for each $A \in \mathcal{A}$ and each $C \in y,\left\{s \in A \cap B: \lambda_{s}^{\prime}(y) \in c \ell C\right\} \neq \emptyset$.

Let $D=\mathcal{A} \times y$, and direct $D$ by agreeing that $(A, C) \leq\left(A^{\prime}, C^{\prime}\right)$ if and only if $A^{\prime} \subseteq A$ and $C^{\prime} \subseteq C$. For $(A, C) \in D$, pick a point $b_{(A, C)} \in A \cap B$ such that $b_{(A, C)}+y \in c l C$. Let $p$ be a limit point of the net $\left\langle b_{(A, C)}\right\rangle_{(A, C) \in D}$. Since each $b_{(A, C)} \in B, p \in c \ell B$. Since $p$ is in every clopen neighborhood of $L, p \in L$. Since $p+y$ is in every clopen neighborhood of $p, p+y=y$.

Since $p \in L \subseteq K(\beta S)$, pick a minimal right ideal $R$ of $\beta S$ such that $p \in R$. Then $y=p+y \in p+\beta S=R$, so $p$ and $y$ are in the group $R \cap L$. Since $p=p+y, p$ is an idempotent.

We shall use the statements in the following theorem to define very strongly central sets. Notice the similarity between statement (a) and Furstenberg's original definition of central.

Theorem 2.9. Let $S$ be a semigroup, and let $M \subseteq S$. The following statements are equivalent.

(a) There exist a minimal dynamical system $\left(X,\left\langle T_{s}\right\rangle_{s \in S}\right)$ an open subset $U$ of $X$ and a point $y \in c \ell U$ such that $\left\{s \in S: T_{s}(y) \in U\right\}=M$.

(b) For every minimal left ideal $L$ of $\beta S$ there exist an open subset $V$ of $L$ and a point $p \in c \ell V$ such that $\{s \in S: s+p \in V\}=M$.

(c) There exist a minimal left ideal $L$ of $\beta S$, an open subset $V$ of $L$, and a point $p \in c \ell V$ such that $\{s \in S: s+p \in V\}=M$.

(d) For every minimal left ideal $L$ of $\beta S$ there exist an open subset $V$ of $L$ and an idempotent $q \in c \ell V$ such that $\{s \in S: s+q \in V\} \in q$ and $\{s \in S: s+q \in V\}=M$. 
(e) There exist a minimal left ideal $L$ of $\beta S$, an open subset $V$ of $L$, and an idempotent $q \in c \ell V$ such that $\{s \in S: s+q \in V\} \in q$ and $\{s \in S: s+q \in$ $V\}=M$.

(f) There is a minimal idempotent $q$ in $\beta S$ such that $M \in q$ and for all $a \in M$ and all $x \in \beta S$, if $a+q=x+q$, then $M \in x$.

Proof. By [15, Lemma 19.6], if $L$ is a minimal left ideal of $\beta S$ and for $s \in S, \lambda_{s}^{\prime}$ is the restriction of $\lambda_{s}$ to $L$, then $\left(L,\left\langle\lambda_{s}^{\prime}\right\rangle_{s \in S}\right)$ is a minimal dynamical system, so $(c) \Rightarrow(a)$. The implications $(d) \Rightarrow(e),(d) \Rightarrow(b),(e) \Rightarrow(c)$, and $(b) \Rightarrow(c)$ are all trivial. We shall show that $(a) \Rightarrow((e)$ and $(f))$ and $(f) \Rightarrow(c)$.

To see that $(a) \Rightarrow((e)$ and $(f))$, assume that (a) holds, and let $L$ be a minimal left ideal of $\beta S$. (For the proof of (e) we are taking $L$ to be arbitrary. For the proof of (f) we are using the fact that $\beta S$ has a minimal left ideal.)

For $s \in S$, let $\lambda_{s}^{\prime}$ be the restriction of $\lambda_{s}$ to $L$. By [15, Theorem 19.8] there is a continuous surjective $\varphi: L \rightarrow X$ such that for all $s \in S, T_{s} \circ \varphi=\varphi \circ \lambda_{s}^{\prime}$.

Let $C=c \ell \varphi^{-1}[U]$. Then $\varphi[C]$ is a closed subset of $X$ containing $U$ so $c \ell U \subseteq \varphi[C]$ and therefore $y \in \varphi[C]$. Pick $p \in C$ such that $y=\varphi(p)$. Let $T=\varphi^{-1}[U]$. Then $T$ is an open subset of $L$ and $p \in c \ell T$.

Let $q$ be the identity of $L \cap(p+\beta S)$. (By [15, Theorem 2.9], $p+\beta S$ is a minimal right ideal of $\beta S$ so by [15, Theorem $2.7(\mathrm{~d})], L \cap(p+\beta S)$ is a group.) Let $\tau$ be the restriction of $\rho_{p}$ to $L$. By [15, Theorem 2.11(c)], $\tau$ is a homeomorphism from $L$ onto $L$. Let $V=\tau^{-1}[T]$. Then $V$ is open in $L$ and $q \in c \ell V$.

We claim that for every $A \subseteq S$ such that $L \subseteq c \ell A, A+q$ is dense in $L$. To see this, let $u \in L$, and let $B \in u$. We claim that $c \ell B \cap(A+q) \neq \emptyset$. Since $L=L+q$, pick $v \in L$ such that $u=v+q$. Then $\{x \in S:-x+B \in q\} \in v$. Also $A \in v$ so pick $x \in A$ such that $-x+B \in q$. Then $x+q \in(A+q) \cap c \ell B$.

Let $D=\{(A, W): A \subseteq S, L \subseteq c \ell A$, and $W$ is an open neighborhood of $q$ in $L\}$. For $(A, W) \in D$, we have that $W \cap V$ is a nonempty open subset of $L$ so $W \cap V \cap$ $(A+q) \neq \emptyset$. Pick $s_{(A, W)} \in A$ such that $s_{(A, W)}+q \in W \cap V$. Direct $D$ by agreeing that $(A, W) \leq\left(A^{\prime}, W^{\prime}\right)$ if and only if $A^{\prime} \subseteq A$ and $W^{\prime} \subseteq W$, and let $v$ be a cluster point of the net $\left\langle s_{(A, W)}\right\rangle_{(A, W) \in D}$ in $\beta S$. Then $v \in c \ell A$ for every $A \subseteq S$ for which $L \subseteq c \ell A$, so since $L=\bigcap\{c \ell A: A \subseteq S$ and $L \subseteq c \ell A\}, v \in L$. Also $v+q \in c \ell W$ for every open neighborhood $W$ of $q$, so $v+q=q$.

Since $v+q=q$, we have that $v$ and $q$ are in the same minimal right ideal. Since also $v \in L$, we have that $v$ is in the group $L \cap(v+\beta S)$, of which $q$ is the identity, and so $v=q$. That is, we have that the net $\left\langle s_{(A, W)}\right\rangle_{(A, W) \in D}$ clusters at $q$. (In fact, we have just shown that it converges to $q$.)

Let $B=\{s \in S: s+q \in V\}$. For each $(A, W) \in D, s_{(A, W)} \in B$, so $q \in c \ell B$, and thus $B \in q$. To complete the proof, we show that $B=\left\{s \in S: T_{s}(y) \in U\right\}=M$. Let $s \in S$. Then

$$
T_{s}(y)=T_{s}(\varphi(p))=\varphi\left(\lambda_{s}^{\prime}(p)\right)=\varphi(s+p)=\varphi(s+q+p)=(\varphi \circ \tau)(s+q) .
$$

Since $V=(\varphi \circ \tau)^{-1}[U]$, we have that $T_{s}(y) \in U$ if and only if $s+q \in V$. Thus (e) has been established.

To complete the proof of (f), let $b \in B$ and $x \in \beta S$, and assume that $b+q=x+q$. Then $x+q \in V$ which is open in $L$, so pick $A \subseteq S$ such that $x+q \in c \ell A$ and $c \ell A \cap L \subseteq V$. Then $\{s \in S:-s+A \in q\} \in x$, so it suffices to show that $\{s \in S:-s+A \in q\} \subseteq B$. Let $s \in S$ be such that $-s+A \in q$. Then $s+q \in c \ell A \cap L$ so $s+q \in V$ and thus $s \in B$. 
Finally we show that $(f) \Rightarrow(c)$, so pick $q$ as guaranteed by (f). Let $L=\beta S+q$, and let $V=L \backslash(c \ell(S \backslash M)+q)$. Then $V$ is open since $c \ell(S \backslash M)+q$ is the continuous image of a compact set. We claim that $M+q \subseteq V$. To this end, let $s \in M$, and suppose that $s+q \notin V$. Then $s+q=x+q$ for some $x \in c \ell(S \backslash M)$. But by our assumption, $M \in x$, a contradiction. Since $M+q \subseteq V$, we have that $\{s \in S: s+q \in$ $V\}=M$. To see that $q \in c \ell V$, let $A \in q$. Then $\{s \in S:-s+A \in q\} \in q$, since $q$ is an idempotent, so pick $s \in M$ such that $-s+A \in q$. Then $s+q \in c \ell A \cap(M+q)$, and so $c \ell A \cap V \neq \emptyset$.

The set of sets satifying the conditions of Theorem 2.9 is not closed under passage to supersets. (Clearly, for $S=\mathbb{N}, 2 \mathbb{N}$ satisfies these conditions - any minimal idempotent will satisfy statement (f). However $2 \mathbb{N} \cup\{1\}$ does not satisfy these statements - if $q$ is any idempotent, then $1+q=(1+q)+q$, but $2 \mathbb{N} \cup\{1\} \notin 1+q$.) Since we want very strongly central to be a reasonable notion of largeness, we define it so that it is closed under passage to supersets. (We will show in Theorem 2.17 that this notion is strictly stronger than strongly central.)

Definition 2.10. Let $S$ be a discrete semigroup. A set $A \subseteq S$ is very strongly central if and only if there is a set $M \subseteq S$ which satisfies (any of) the statements of Theorem 2.9 with $M \subseteq A$.

Theorem 2.11. Let $S$ be a discrete semigroup, and let $A$ be a very strongly central subset of $S$. Then there is a minimal right ideal $R$ of $\beta S$ such that $A$ is a member of every idempotent in $R$. In particular, $A$ is strongly central.

Proof. Pick $M \subseteq A$ and a minimal idempotent $q$ in $\beta S$ such that for all $a \in M$ and all $x \in \beta S$, if $a+q=x+q$, then $M \in x$. Let $R=q+\beta S$. Then $R$ is a minimal right ideal of $\beta S$. Let $p$ be an idempotent in $R$. Then $R=p+\beta S$. Thus $q+p=p$ and $p+q=q$. Therefore, for every $a \in M, a+p+q=a+q$, so for every $a \in M$, $M \in a+p$. Therefore $M \subseteq\{s \in S:-s+M \in p\}$ so $M \in q+p=p$.

For the "in particular" conclusion, let $L$ be a minimal left ideal of $\beta S$, and pick an idempotent $p \in L \cap R$.

Question 2.12. Let $S$ be a discrete semigroup, and let $A$ be a subset of $S$ such that there is some minimal right ideal $R$ of $\beta S$ with the property that $A$ is a member of each idempotent in $R$. Must $A$ be very strongly central?

Finally, we turn our attention to strongly central subsets of $\mathbb{N}$. It is a consequence of [14, Corollary 2.13] that any central subset of $\mathbb{N}$ contains countably many pairwise disjoint central subsets of $\mathbb{N}$.

Theorem 2.13. There exist infinitely many pairwise disjoint very strongly central subsets of $\mathbb{N}$.

Proof. Let $X=\mathbb{R} / \mathbb{Z}$, identify $X$ with $\left[-\frac{1}{2}, \frac{1}{2}\right)$ and let $\alpha$ be an irrational number. Define $T: X \rightarrow X$ by $T(x)=w(x+\alpha)$. Then, as is well known, $\left(X,\left\langle T^{n}\right\rangle_{n \in \mathbb{N}}\right)$ is a minimal dynamical system. Choose a sequence $\left\langle U_{n}\right\rangle_{n=1}^{\infty}$ of pairwise disjoint open subsets of $X$ with $0 \in c \ell U_{n}$ for each $n$. Then by Theorem [2.9, for each $n$, $\left\{x \in \mathbb{N}: T_{x}(0) \in U_{n}\right\}$ is very strongly central.

Question 2.14. Let $A$ be a strongly central subset of $\mathbb{N}$. Must $A$ contain infinitely many pairwise disjoint strongly central sets? 
It is a consequence of [9, Theorem 3.3] that any central subset of $\mathbb{N}$ contains $\mathfrak{c}$ almost disjoint central subsets of $\mathbb{N}$. (Two subsets of $\mathbb{N}$ are almost disjoint if and only if their intersection is finite.) However, by [9, Corollary 4.2], there does not exist an uncountable family of almost disjoint syndetic subsets of $\mathbb{N}$ so there does not exist an uncountable family of almost disjoint strongly central subsets of $\mathbb{N}$.

We have already observed that strongly central sets are syndetic and syndetic sets are translation invariant, so every translation of a strongly central set is syndetic. We see now that the converse fails. (Recall by way of contrast that every piecewise syndetic set is a translate of a central set.)

For $x \in \mathbb{N}$ define $\operatorname{supp}(x) \subseteq \mathbb{N} \cup\{0\}$ by $x=\sum_{t \in \operatorname{supp}(x)} 2^{t}$. Let $\mathbb{H}=\bigcap_{n=1}^{\infty} c \ell\left(2^{n} \mathbb{N}\right)$.

Theorem 2.15. There exists a syndetic subset $C$ of $\mathbb{N}$ such that for all $x \in \mathbb{Z}$, $(x+C) \cap \mathbb{N}$ is not strongly central in $\mathbb{N}$.

Proof. Let $A=\{x \in \mathbb{N}: \max \operatorname{supp}(x)$ is even $\}$ and $B=\{x \in \mathbb{N}: \max \operatorname{supp}(x)$ is odd . We observe that if $q \in c \ell A \cap \mathbb{H}$ and $x \in \mathbb{N}$, then $x+q \in c \ell A$. Also if $q \in c \ell A \cap \mathbb{H},\left\{2^{n}: n \in \mathbb{N}\right\} \notin q$, and $x \in \mathbb{N}$, then $-x+q \in c \ell A$. (In the latter case, the addition is taking place in $\beta \mathbb{Z}$.) The identical statements apply with $B$ replacing $A$ throughout.

Let $C=(A \cap(2 \mathbb{N}-1)) \cup(B \cap 2 \mathbb{N})$. Then $C$ is syndetic - in fact $C$ has no gaps longer than length 2. Suppose we have some $x \in \mathbb{Z}$ such that $x+C$ is strongly central.

Suppose first that $x$ is odd. Pick $q \in c \ell B \cap \mathbb{H}$. Then $\mathbb{N}+q \subseteq c \ell B$ so $\beta \mathbb{N}+q \subseteq c \ell B$. Pick a minimal left ideal $L$ of $\beta \mathbb{N}$ such that $L \subseteq \beta \mathbb{N}+q$ and pick an idempotent $p \in L \cap c \ell(x+C)$. Note that $\left\{2^{n}: n \in \mathbb{N}\right\} \notin p$, in fact $\left\{2^{n}: n \in \mathbb{N}\right\}$ is not a member of any idempotent. Now either $x+(A \cap(2 \mathbb{N}-1)) \in p$ or $x+(B \cap 2 \mathbb{N}) \in p$. In the first case $A \in-x+p$ while $-x+p \in c \ell B$. In the second case $2 \mathbb{N} \in-x+p$ while $x$ is odd and $2 \mathbb{N} \in p$. In either case we have a contradiction.

We must then have that $x$ is even. Pick $q \in c \ell A \cap \mathbb{H}$. Then $\mathbb{N}+q \subseteq c \ell A$ so $\beta \mathbb{N}+q \subseteq c \ell A$. Pick a minimal left ideal $L$ of $\beta \mathbb{N}$ such that $L \subseteq \beta \mathbb{N}+q$ and pick an idempotent $p \in L \cap c \ell(x+C)$. Again we have that $\left\{2^{n}: n \in \mathbb{N}\right\} \notin p$. Now either $x+(A \cap(2 \mathbb{N}-1)) \in p$ or $x+(B \cap 2 \mathbb{N}) \in p$. In the first case $2 \mathbb{N}-1 \in-x+p$ while $x$ is even and $2 \mathbb{N} \in p$. In the second case $B \in-x+p$ while $-x+p \in c \ell A$. In either case we have a contradiction.

Corollary 2.16. There exists a syndetic subset $D$ of $\mathbb{Z}$ such that for all $x \in \mathbb{Z}$, $x+D$ is not strongly central in $\mathbb{Z}$.

Proof. Pick $C$ as guaranteed by Theorem 2.15. Let $D=C \cup-C$. It is routine to verify that $D$ is syndetic in $\mathbb{Z}$. Let $x \in \mathbb{Z}$ and suppose that $x+D$ is strongly central in $\mathbb{Z}$. Since $(x+C) \cap \mathbb{N}$ is not strongly central in $\mathbb{N}$, pick a minimal left ideal $L$ of $\beta \mathbb{N}$ such that $(x+C) \cap \mathbb{N}$ is not a member of any idempotent in $L$. By [15, Theorem 4.37], $L$ is a minimal left ideal of $\mathbb{N}^{*}=\beta \mathbb{N} \backslash \mathbb{N}$ and by [15, Exercise 4.3.5], $\mathbb{N}^{*}$ is a left ideal of $\beta \mathbb{Z}$, so by [15, Lemma 1.43], $L$ is a minimal left ideal of $\beta \mathbb{Z}$. It thus suffices to show that $x+D$ is not a member of an idempotent in $L$. Suppose instead we have an idempotent $p \in L$ such that $x+D \in p$ and thus $(x+D) \cap \mathbb{N} \in p$. We then have that $((x+D) \cap \mathbb{N}) \backslash((x+C) \cap \mathbb{N}) \in p$. This is a contradiction since $((x+D) \cap \mathbb{N}) \backslash((x+C) \cap \mathbb{N})$ is finite.

We see now that the notion of very strongly central is strictly stronger than the notion of strongly central. 
Theorem 2.17. Let $A=\{x \in \mathbb{N}: \min \operatorname{supp}(x) \equiv \max \operatorname{supp}(x)(\bmod 2)\}$. Then $A$ is strongly central but not very strongly central.

Proof. For $i \in\{0,1\}$ let $B_{i}=\{x \in \mathbb{N}: \min \operatorname{supp}(x) \equiv i(\bmod 2)\}$ and let $C_{i}=\{x \in$ $\mathbb{N}: \max \operatorname{supp}(x) \equiv i(\bmod 2)\}$. For each $i \in\{0,1\}, \overline{B_{i}} \cap \mathbb{H}$ is a right ideal of $\mathbb{H}$, so any minimal right ideal of $\mathbb{H}$ is contained in $\overline{B_{i}} \cap \mathbb{H}$ for some $i \in\{0,1\}$. Similarly, for each $i \in\{0,1\}, \overline{C_{i}} \cap \mathbb{H}$ is a left ideal of $\mathbb{H}$, so any minimal left ideal of $\mathbb{H}$ is contained in $\overline{C_{j}} \cap \mathbb{H}$ for some $j \in\{0,1\}$.

To see that $A$ is strongly central, let $L$ be a left ideal of $\beta \mathbb{N}$ and pick a minimal left ideal $L^{\prime}$ of $\mathbb{H}$ with $L^{\prime} \subseteq L \cap \mathbb{H}$. Pick $i \in\{0,1\}$ such that $L^{\prime} \subseteq \overline{C_{i}} \cap \mathbb{H}$. Pick any idempotent $p \in B_{i} \cap L^{\prime}$. Then $p \in \bar{A} \cap L$.

To see that $A$ is not very strongly central, let $R$ be a right ideal of $\beta \mathbb{N}$. We show that there is an idempotent $q \in R \backslash \bar{A}$ so that, by Theorem 2.11, $A$ is not very strongly central. Pick a minimal right ideal $R^{\prime}$ of $\mathbb{H}$ such that $R^{\prime} \subseteq R \cap \mathbb{H}$, and pick $i \in\{0,1\}$ such that $R^{\prime} \subseteq \overline{B_{i}} \cap \mathbb{H}$. Let $j=i-1$, and pick an idempotent $q \in R^{\prime} \cap \overline{C_{j}}$. Then $q \in R \backslash \bar{A}$.

\section{RETURNS OF POLYNOMIALS NEAR ZERO MOD 1}

This section is devoted to the proof of our main result in this paper, Theorem 3.2 . which was formulated in the introduction. In view of the discussion in Section 2 and in view of the fact that the implications $(a) \Rightarrow(b),(b) \Rightarrow(c)$, and $(c) \Rightarrow(d)$ are all trivial, one only needs to show that (d) implies (e) and (e) implies (a). Also, as can be seen in the proof of Theorem 3.2, the proof that (d) implies (e) is quite easy. So the main difficulty is in establishing the implication $(e) \Rightarrow(a)$.

In view of statement (a) of Theorem 2.9, one is tempted to look for a minimal dynamical system $\left(X,\left\langle T^{n}\right\rangle_{n \in \mathbb{N}}\right)$ such that for some point $y \in X$ and some open subset $W$ of $X$ with $y \in c \ell W,\left\{n \in \mathbb{N}: T^{n}(y) \in W\right\} \subseteq\{n \in \mathbb{N}$ : $\left.\left(w\left(P_{1}(n)\right), w\left(P_{2}(n)\right), \ldots, w\left(P_{v}(n)\right)\right) \in U\right\}$. One can indeed construct a minimal dynamical system with $X=\mathbb{T}^{d}$ for some $d \in \mathbb{N}$ such that some set of $v$ coordinates of the orbit of $(0,0, \ldots, 0) \in \mathbb{T}^{d}$ looks like $\left(w\left(P_{1}(n)\right), w\left(P_{2}(n)\right), \ldots, w\left(P_{v}(n)\right)\right)$, and from this one can derive the desired result.

We, however, choose a somewhat different approach. Namely, we will prove first a rather special-looking case of Theorem 3.2 namely Theorem 3.1, and then show that Theorem 3.2 follows from it. The reason we have chosen this path is two-fold. First, Theorem 3.1 is easy to prove (and we will actually indicate more than one way to do this), and, second, this allows us to stress the somewhat revealing fact that Theorem 3.2 can be derived from this relatively simple special case.

Theorem 3.1. Let $k, l \in \mathbb{N}$ and let $\mu_{1}, \mu_{2}, \ldots, \mu_{l}$ be real numbers for which $1, \mu_{1}$, $\mu_{2}, \ldots, \mu_{l}$ are linearly independent over $\mathbb{Q}$. Let $U$ be an open subset of $\mathbb{T}^{l k}$ for which $0 \in c \ell U$. Then

$$
\begin{aligned}
\{x \in \mathbb{N}: & \left(w\left(\mu_{1} x\right), w\left(\mu_{2} x\right), \ldots, w\left(\mu_{l} x\right)\right. \\
& w\left(\mu_{1} x^{2}\right), w\left(\mu_{2} x^{2}\right), \ldots, w\left(\mu_{l} x^{2}\right), \ldots, \\
& \left.\left.w\left(\mu_{1} x^{k}\right), w\left(\mu_{2} x^{k}\right), \ldots, w\left(\mu_{l} x^{k}\right)\right) \in U\right\}
\end{aligned}
$$

is very strongly central. 
Proof. We show now that there exists a homeomorphism $T: \mathbb{T}^{l k} \rightarrow \mathbb{T}^{l k}$ such that for each $n \in \mathbb{N}, T^{n}(0,0, \ldots, 0)=\left(\mu_{1} n, \mu_{1} n^{2}, \ldots, \mu_{1} n^{k}, \ldots, \mu_{l} n, \mu_{l} n^{2}, \ldots, \mu_{l} n^{k}\right)$. To construct $T$ we first define for each $i \in\{1,2, \ldots, l\}$ the homeomorphism $T_{i}: \mathbb{T}^{k} \rightarrow$ $\mathbb{T}^{k}$ by

$$
\begin{gathered}
T_{i}\left(x_{1}, x_{2}, \ldots, x_{k}\right)=\left(x_{1}+\mu_{i}, 2 x_{1}+x_{2}+\mu_{i}, 3 x_{1}+3 x_{2}+x_{3}+\mu_{i}, \ldots,\right. \\
\left.\left(\begin{array}{c}
k \\
1
\end{array}\right) x_{1}+\left(\begin{array}{l}
k \\
2
\end{array}\right) x_{2}+\ldots+\left(\begin{array}{l}
k \\
k
\end{array}\right) x_{k}+\mu_{i}\right) .
\end{gathered}
$$

By an easy induction one has that for each $n \in \mathbb{N}$,

$$
T_{i}^{n}(0,0, \ldots, 0)=\left(\mu_{i} n, \mu_{i} n^{2}, \ldots, \mu_{i} n^{k}\right) .
$$

Now let $T=T_{1} \times \ldots \times T_{l}$. That is, $T: \mathbb{T}^{l k} \rightarrow \mathbb{T}^{l k}$ is defined as

$$
\begin{aligned}
T\left(x_{1}, x_{2}, \ldots, x_{l k}\right)= & T_{1}\left(x_{1}, x_{2}, \ldots, x_{k}\right) \frown T_{2}\left(x_{k+1}, x_{k+2} \ldots, x_{2 k}\right) \\
& \ldots \frown T_{l}\left(x_{(l-1) k+1}, x_{(l-1) k+2}, \ldots, x_{l k}\right),
\end{aligned}
$$

where $\frown$ denotes concatenation so that, for example, $(1,3,2) \frown(2,1)=(1,3,2,2,1)$.

As we remarked earlier, by statement (a) of Theorem 2.9. to complete the proof, it suffices to show that the dynamical system $\left(\mathbb{T}^{l k},\left\langle T^{n}\right\rangle_{n \in \mathbb{N}}\right)$ is minimal. By [13, Theorem 1.011], $\left\{T^{n}(\overline{0}): n \in \mathbb{N}\right\}$ is dense in $\mathbb{T}^{l k}$ so by [12, Theorem 1.17] it suffices to show that $\overline{0}=(0,0, \ldots, 0)$ is a uniformly recurrent point of $\left(\mathbb{T}^{l k},\left\langle T^{n}\right\rangle_{n \in \mathbb{N}}\right)$. To this end, let $V$ be a neighborhood of $\overline{0}$, and let $A=\left\{n \in \mathbb{N}: T^{n}(\overline{0}) \in V\right\}$. By [12, Theorem 1.21], the set $A$ is syndetic in $\mathbb{Z}$ and therefore in $\mathbb{N}$ so that $\overline{0}$ is uniformly recurrent, as claimed.

We now indicate other ways to complete the proof of Theorem 3.1 using classical results. We observe that, as is well known, if $i \in\{1,2, \ldots, l\}$ (so $\mu_{i} \notin \mathbb{Q}$ ), then $T_{i}$ is minimal and distal (i.e., for all $x, y \in \mathbb{T}^{k}$, if the infinum of the distances between $T^{n}(x)$ and $T^{n}(y)$ as $n$ ranges over $\mathbb{N}$ is 0 , then $x=y$ ). The property of distality is obvious from the definition of $T_{i}$. As for the minimality, it may be proved in a number of ways. For example, it follows from [12, Lemma 1.25]. Another way to see that $T_{i}$ is minimal is to invoke the simple fact [1, Corollary 7, p. 69] that a distal flow is minimal if and only if there is a point with dense orbit. The fact that in $\left(\mathbb{T}^{k},\left\langle T_{i}^{n}\right\rangle_{n \in \mathbb{N}}\right)$ one has a point with dense orbit follows immediately from the fact that for each $n \in \mathbb{N}, T_{i}^{n}(0,0, \ldots, 0)=\left(\mu_{i} n, \mu_{i} n^{2}, \ldots, \mu_{i} n^{k}\right)$ and that the sequence $\left\langle\left(\mu_{i} n, \mu_{i} n^{2}, \ldots, \mu_{i} n^{k}\right)\right\rangle$ is dense, indeed, uniformly distributed, in $\mathbb{T}^{k}$. The denseness was established by Hardy and Littlewood in the result cited above, and the uniform distribution was proved by Weyl [20. Also see Section 1.6 and especially exercise 6.8 of [17. See also [12, Theorem 1.26, and Paragraph 3 in Chapter 3].

Note that since $1, \mu_{1}, \ldots, \mu_{l}$ are linearly independent over $\mathbb{Q}, T$ is a minimal transformation. Again, this can be seen in a variety of ways. In particular, each of the following three possibilities works:

(1) It is a corollary of an appropriately modified version of [12, Lemma 1.25].

(2) By using [13, Theorem 1.011].

(3) By applying Weil's criterion in the form of [17, Theorem 6.3].

We are now ready to prove the main theorem. 
Theorem 3.2. Let $v \in \mathbb{N}$ and for $u \in\{1,2, \ldots, v\}$, let $P_{u}$ be a polynomial with real coefficients and zero constant term. The following statements are equivalent.

(a) Whenever $U$ is an open subset of $\mathbb{T}^{v}$ with $\overline{0} \in c \ell U$, $\left\{n \in \mathbb{N}:\left(w\left(P_{1}(n)\right), w\left(P_{2}(n)\right), \ldots, w\left(P_{v}(n)\right)\right) \in U\right\}$ is very strongly central.

(b) Whenever $U$ is an open subset of $\mathbb{T}^{v}$ with $\overline{0} \in c \ell U$, $\left\{n \in \mathbb{N}:\left(w\left(P_{1}(n)\right), w\left(P_{2}(n)\right), \ldots, w\left(P_{v}(n)\right)\right) \in U\right\}$ is strongly central.

(c) Whenever $U$ is an open subset of $\mathbb{T}^{v}$ with $\overline{0} \in \mathrm{clU}$, $\left\{n \in \mathbb{N}:\left(w\left(P_{1}(n)\right), w\left(P_{2}(n)\right), \ldots, w\left(P_{v}(n)\right)\right) \in U\right\}$ is central.

(d) Whenever $U$ is an open subset of $\mathbb{T}^{v}$ with $\overline{0} \in c \ell U$, $\left\{n \in \mathbb{N}:\left(w\left(P_{1}(n)\right), w\left(P_{2}(n)\right), \ldots, w\left(P_{v}(n)\right)\right) \in U\right\} \neq \emptyset$.

(e) Any nontrivial linear combination of $\left\{P_{u}: u \in\{1,2, \ldots, v\}\right\}$ over $\mathbb{Q}$ has at least one irrational coefficient.

Proof. That (a) implies (b), (b) implies (c), and (c) implies (d) is trivial.

To see that (d) implies (e) assume that (e) does not hold. Then there are integers, $h_{1}, h_{2}, \ldots, h_{v}$, not all zero, such that all the coefficients of $h_{1} P_{1}+h_{2} P_{2}+\ldots+h_{v} P_{v}$ are integers. So $w\left(h_{1} P_{1}(n)+h_{2} P_{2}(n)+\ldots+h_{v} P_{v}(n)\right)=0$ for every $n \in \mathbb{N}$. Now $\tau: \mathbb{T}^{v} \rightarrow \mathbb{T}$ defined by $\tau\left(t_{1}, t_{2}, \ldots, t_{v}\right)=h_{1} t_{1}+h_{2} t_{2}+\ldots+h_{v} t_{v}$ is continuous. Let $U=\left\{\vec{t} \in \mathbb{T}^{v}: \tau(\vec{t}) \neq 0\right\}$. Then $U$ is an open subset of $\mathbb{T}^{v}$ for which $\overline{0} \in \operatorname{cl}(U)$. So (d) does not hold.

To see that (e) implies (a), assume that any nontrivial linear combination of $\left\{P_{u}\right.$ : $u \in\{1,2, \ldots, v\}\}$ over $\mathbb{Q}$ has at least one irrational coefficient, and let $U$ be an open subset of $\mathbb{T}^{v}$ with $\overline{0} \in c \ell U$. Pick $k \in \mathbb{N}$ and for $u \in\{1,2, \ldots, v\}$, and pick $\left\langle a_{u, s}\right\rangle_{s=1}^{k}$ in $\mathbb{R}$ such that for each $x, P_{u}(x)=\sum_{s=1}^{k} a_{u, s} x^{t}$. Let $1, \mu_{1}, \mu_{2}, \ldots, \mu_{l}$ be linearly independent over $\mathbb{Q}$ such that $\left\{a_{u, s}: u \in\{1,2, \ldots, v\}\right.$ and $\left.s \in\{1,2, \ldots, k\}\right\}$ is contained in the rational linear span of $\left\{1, \mu_{1}, \ldots, \mu_{l}\right\}$. For $u \in\{1,2, \ldots, u\}$ and $t \in$ $\{1,2, \ldots, k\}$, let $\langle\alpha(u, s, i)\rangle_{i \in L}$ be the rational numbers such that $a_{u, s}=\alpha(u, s, 0)+$ $\sum_{i=1}^{l} \alpha(u, s, i) \mu_{i}$. Then for each $u \in\{1,2, \ldots, v\}$ and $x \in \mathbb{N}$,

$$
P_{u}(x)=Q_{u}(x)+\sum_{i=1}^{l} \sum_{s=1}^{k} \alpha(u, s, i) \mu_{i} x^{s}
$$

where $Q_{u}(x)=\sum_{s=1}^{k} \alpha(u, s, 0) x^{s}$.

Let $M$ be the $v \times l k$ matrix

$$
\left(\begin{array}{cccccccccc}
\alpha(1,1,1) & \ldots & \alpha(1,1, l) & \alpha(1,2,1) & \ldots & \alpha(1,2, l) & \ldots & \alpha(1, k, 1) & \ldots & \alpha(1, k, l) \\
\alpha(2,1,1) & \ldots & \alpha(2,1, l) & \alpha(2,2,1) & \ldots & \alpha(2,2, l) & \ldots & \alpha(2, k, 1) & \ldots & \alpha(2, k, l) \\
\vdots & \ddots & \vdots & \vdots & \ddots & \vdots & \ddots & \vdots & \ddots & \vdots \\
\alpha(v, 1,1) & \ldots & \alpha(v, 1, l) & \alpha(v, 2,1) & \ldots & \alpha(v, 2, l) & \ldots & \alpha(v, k, 1) & \ldots & \alpha(v, k, l)
\end{array}\right)
$$

Then $M$ has rank $v$. (If not, then there exist $\left(\gamma_{1}, \gamma_{2}, \ldots, \gamma_{v}\right) \in \mathbb{Q}^{v} \backslash\{\overline{0}\}$ such that for each $t \in\{1,2, \ldots, k\}$ and $i \in\{1,2, \ldots, l\}, \sum_{u=1}^{v} \gamma_{u} \alpha(u, t, i)=0$ and consequently, $\sum_{u=1}^{v} \gamma_{u} P_{u}=\sum_{u=1}^{v} Q_{u}$, a polynomial with all rational coefficients.)

We first consider the special case in which all the entries of $M$ as well as all of the numbers $\alpha(u, s, 0)$ are integers. We regard the elements of $\mathbb{R}^{l k}$ and $\mathbb{T}^{l k}$ as column vectors, so that $M \vec{x}$ is defined if $\vec{x} \in \mathbb{R}^{l k}$ or if $\vec{x} \in \mathbb{T}^{l k}$. Further recall that we are taking $\mathbb{T}=\left[-\frac{1}{2}, \frac{1}{2}\right)$, so in particular $\left(-\frac{1}{2}, \frac{1}{2}\right) \subseteq \mathbb{T}$. Let $\phi: \mathbb{T}^{l k} \rightarrow \mathbb{T}^{v}$ and $L: \mathbb{R}^{l k} \rightarrow \mathbb{R}^{v}$ be the functions defined by $\phi(\vec{x})=M \vec{x}$ and $L(\vec{x})=M \vec{x}$, where in 
the first case the computations are done in $\mathbb{T}$ and in the second they are done in $\mathbb{R}$. Pick $\delta>0$ such that for all $\vec{x} \in(-\delta, \delta)^{l k}, \phi(\vec{x})=L(\vec{x})$. Since the rank of $M$ is $v$, we have that $L$ is surjective, and therefore by the Open Mapping Theorem, $L$ is an open map. (One may verify this instance of the Open Mapping Theorem as follows. Let $V_{1}=\left\{x \in \mathbb{R}^{l k}: M x=0\right\}$. Then there is a vector subspace $V_{2}$ of $\mathbb{R}^{l k}$ such that $\mathbb{R}^{l k}=V_{1} \oplus V_{2}$ and the restriction of $M$ to $V_{2}$ is an isometry between $V_{2}$ and $\mathbb{R}^{v}$. It is then obvious that $M$ is an open map.)

We claim that $\overline{0} \in c \ell \phi^{-1}[U]$. To this end, let $W$ be an open neighborhood of $\overrightarrow{0}$ in $\mathbb{T}^{l k}$ with $W \subseteq(-\delta, \delta)^{l k}$. Then $W$ is an open neighborhood of $\overrightarrow{0}$ in $\mathbb{R}^{l k}$ so $L[W]$ is an open neighborhood of $\overline{0}$ in $\mathbb{R}^{v}$ and thus also in $\mathbb{T}^{v}$. Pick $\vec{x} \in \mathbb{T}^{l k}$ such that $L(\vec{x}) \in U$. Then $\vec{x} \in W \cap \phi^{-1}[U]$. Let

$$
B=\left\{n \in \mathbb{N}:\left(w\left(\mu_{1} n\right), w\left(\mu_{2} n\right), \ldots, w\left(\mu_{l} n^{k}\right)\right) \in \phi^{-1}[U] \cap(-\delta, \delta)^{l k}\right\} .
$$

Then by Theorem 3.1, $B$ is very strongly central. We claim that

$$
B \subseteq\left\{n \in \mathbb{N}:\left(w\left(P_{1}(n)\right), w\left(P_{2}(n)\right), \ldots, w\left(P_{v}(n)\right)\right) \in U\right\} .
$$

To this end let $n \in B$. Then $L\left(w\left(\mu_{1} n\right), w\left(\mu_{2} n\right), \ldots, w\left(\mu_{l} n^{k}\right)\right) \in U$ and

$$
L\left(w\left(\mu_{1} n\right), w\left(\mu_{2} n\right), \ldots, w\left(\mu_{l} n^{k}\right)\right)=M\left(\begin{array}{c}
w\left(\mu_{1} n\right) \\
w\left(\mu_{2} n\right) \\
\vdots \\
w\left(\mu_{l} n^{k}\right)
\end{array}\right)=\left(\begin{array}{c}
w\left(P_{1}(n)\right) \\
w\left(P_{2}(n)\right) \\
\vdots \\
w\left(P_{v}(n)\right)
\end{array}\right)
$$

because $w\left(Q_{u}(n)\right)=0$ for all $u \in\{1,2, \ldots, v\}$. This establishes (a) in the special case in which all the numbers $\alpha(u, s, i)$ are integers.

To establish the general case, choose $d \in \mathbb{N}$ for which all the numbers $d \alpha(u, s, i)$ are integers. Since $d M$ is the matrix corresponding to the polynomials $d P_{1}, d P_{2}, \ldots$, $d P_{v}$, it follows from the special case considered that

$$
\left\{n \in \mathbb{N}:\left(w\left(d P_{1}(n)\right), w\left(d P_{2}(n)\right), \ldots, w\left(d P_{v}(n)\right)\right) \in d\left(U \cap\left(-\frac{1}{2 d}, \frac{1}{2 d}\right)^{v}\right)\right\}
$$

is very strongly central. Since multiplication by $d$ is an injective map on the subset $\left(-\frac{1}{2 d}, \frac{1}{2 d}\right)^{v}$ of $\mathbb{T}^{v}$, the proof is complete.

Corollary 3.3. Suppose that $P_{1}, P_{2}, \ldots, P_{v}$ are polynomials with real coefficients and zero constant term and that any nontrivial linear combination of these polynomials over $\mathbb{Q}$ has at least one irrational coefficient. Let $G$ be the set of real numbers c such that

$\left(^{*}\right)$ whenever $U$ is an open subset of $\mathbb{T}^{v}$ for which

$$
\left(w\left(P_{1}(c)\right), w\left(P_{2}(c)\right), \ldots, w\left(P_{v}(c)\right)\right) \in c \ell U,
$$

one has that $\left\{n \in \mathbb{N}:\left(w\left(P_{1}(n+c)\right), w\left(P_{2}(n+c)\right), \ldots, w\left(P_{v}(n+c)\right)\right) \in U\right\}$ is very strongly central.

Then $\mathbb{Q} \subseteq G$ and $\mathbb{R} \backslash G$ is countable.

Proof. For $c \in \mathbb{R}$ and $u \in\{1,2, \ldots, v\}$, let $Q_{c, u}(x)=P_{u}(x+c)-P_{u}(c)$ and note that $Q_{c, u}$ is a real polynomial with zero constant term. We claim that to show that $c \in G$, it suffices to show that any nontrivial linear combination of $\left\{Q_{c, u}: u \in\right.$ $\{1,2, \ldots, v\}\}$ over $\mathbb{Q}$ has at least one irrational coefficient. Assume we have done so. Let $U$ be an open subset of $\mathbb{T}^{v}$ for which $\left(w\left(P_{1}(c)\right), w\left(P_{2}(c)\right), \ldots, w\left(P_{v}(c)\right)\right) \in c \ell U$, and let $V=-\left(w\left(P_{1}(c)\right), w\left(P_{2}(c)\right), \ldots, w\left(P_{v}(c)\right)\right)+U$. Then $\overline{0} \in c \ell V$, so by 
Theorem 3.2. $\left\{n \in \mathbb{N}:\left(w\left(P_{1}(n+c)\right), w\left(P_{2}(n+c)\right), \ldots, w\left(P_{v}(n+c)\right)\right) \in U\right\}=$ $\left\{n \in \mathbb{N}:\left(w\left(Q_{c, 1}(n)\right), w\left(Q_{c, 2}(n)\right), \ldots, w\left(Q_{c, v}(n)\right)\right) \in V\right\}$ is very strongly central. Thus the claim is established.

Let $k=\max \left\{\operatorname{deg}\left(P_{u}\right): u \in\{1,2, \ldots, v\}\right\}$ and for each $u \in\{1,2, \ldots, v\}$, let $\left\langle a_{u, s}\right\rangle_{s=1}^{k}$ be such that $P_{u}(x)=\sum_{s=1}^{k} a_{u, s} x^{s}$.

To see that $\mathbb{Q} \subseteq G$, let $c \in \mathbb{Q}$ be given and let $\left\langle b_{u, s}\right\rangle_{s=1}^{k}$ be such that $Q_{c, u}(x)=$ $\sum_{s=1}^{k} b_{u, s} x^{s}$. Let $A$ and $B$ be the $v \times k$ matrices with entries $a_{u, s}$ and $b_{u, s}$ respectively. Let

$$
C=\left(\begin{array}{ccccc}
1 & 0 & 0 & \ldots & 0 \\
2 c & 1 & 0 & \ldots & 0 \\
3 c^{2} & 3 c & 1 & \ldots & 0 \\
\vdots & \vdots & \vdots & \ddots & \vdots \\
\left(\begin{array}{l}
k \\
1
\end{array}\right) c^{k-1} & \left(\begin{array}{l}
k \\
2
\end{array}\right) c^{k-2} & \left(\begin{array}{l}
k \\
3
\end{array}\right) c^{k-3} & \ldots & 1
\end{array}\right) .
$$

Then $B=A C$ and $C$, being lower triangular, is invertible. Suppose that there is a nontrivial linear combination of $\left\{Q_{c, u}: u \in\{1,2, \ldots, v\}\right\}$ over $\mathbb{Q}$ with no rational coefficients. That is to say, there is a nonzero $1 \times v$ matrix $R$ over $\mathbb{Q}$ such that all entries of $R B$ are rational. But then all entries of $R A=R B C^{-1}$ are rational, while any nontrivial linear combination of $\left\{P_{u}: u \in\{1,2, \ldots, v\}\right\}$ over $\mathbb{Q}$ has at least one irrational coefficient, a contradiction.

To complete the proof, we show that for all except countably many values of $c$ and all $\left(\alpha_{1}, \alpha_{2}, \ldots, \alpha_{v}\right) \in \mathbb{Q}^{v} \backslash\{\overline{0}\}$, the linear coefficient of $\sum_{u=1}^{v} \alpha_{u} Q_{c, u}(x)$ is irrational. For this, it in turn suffices to show that for a given $\left(\alpha_{1}, \alpha_{2}, \ldots, \alpha_{v}\right)$ and all but countably many values of $c$, the linear coefficient of $\sum_{u=1}^{v} \alpha_{u} Q_{c, u}(x)$ is irrational. So let $\left(\alpha_{1}, \alpha_{2}, \ldots, \alpha_{v}\right) \in \mathbb{Q}^{v} \backslash\{\overline{0}\}$ be given. For each $u \in\{1,2, \ldots, v\}$, the linear coefficient of $Q_{c, u}(x)$ is $\sum_{j=1}^{k} a_{u, j} j c^{j-1}$, so the linear coefficient of $\sum_{u=1}^{v} \alpha_{u} Q_{c, u}(x)$ is $\sum_{j=1}^{k} j c^{j-1} \sum_{u=1}^{v} \alpha_{u} a_{u, j}$.

Assume first that for all $j \in\{2,3, \ldots, k\}, \sum_{u=1}^{v} \alpha_{u} a_{u, j}=0$. Then $\sum_{u=1}^{v} \alpha_{u} a_{u, 1}$ is irrational (since $\sum_{u=1}^{v} \alpha_{u} P_{u}(x)$ is a nontrivial linear combination of $\left\{P_{u}: u \in\right.$ $\{1,2, \ldots, v\}\})$ and $\sum_{u=1}^{v} \alpha_{u} a_{u, 1}$ is the linear coefficient of $\sum_{u=1}^{v} \alpha_{u} Q_{c, u}(x)$.

Finally, assume that for some $j \in\{2,3, \ldots, k\}, \sum_{u=1}^{v} \alpha_{u} a_{u, j} \neq 0$. Then

$$
\sum_{j=1}^{k} j c^{j-1} \sum_{u=1}^{v} \alpha_{u} a_{u, j}
$$

is a nonconstant polynomial in $c$. Given any rational number $\delta$, there are at most $k$ solutions to the equation $\sum_{j=1}^{k} j c^{j-1} \sum_{u=1}^{v} \alpha_{u} a_{u, j}=\delta$, so for all but countably many values of $c, \sum_{j=1}^{k} j c^{j-1} \sum_{u=1}^{v} \alpha_{u} a_{u, j}$ is irrational.

We show in the following example that $\mathbb{Q}$ is the largest set that can be guaranteed to be contained in the set $G$ of Corollary 3.3 .

Example 3.4. Let $c$ be irrational and let $P(x)=x^{2}-2 c x$. Then $P(x+c)=$ $x^{2}+P(c)$. Let $U=\mathbb{T} \backslash\{w(P(c))\}$. Then $\{n \in \mathbb{N}: w(P(n+c)) \in U\}=\emptyset$.

In the proof of Theorem 3.1 we used the fact from 12 that the set $A$ is syndetic. In fact, in [12, Theorem 2.19] the much stronger fact that $A$ is $\operatorname{IP}^{*}$ in $\mathbb{N}$ is established. We prove a stronger fact in a more general setting in the following two lemmas, where $\mathbb{R}_{d}$ denotes $\mathbb{R}$ with the discrete topology. 
Lemma 3.5. Let $P$ be a polynomial with real coefficients and zero constant term. Then for every idempotent $p$ in $\left(\beta \mathbb{R}_{d},+\right)$ and every $q \in \beta \mathbb{R}_{d}, \widehat{w \circ P}(p)=0$ and $\widetilde{w \circ P}(q+p)=\widetilde{w \circ P}(q)$, where $\widetilde{w \circ P}: \beta \mathbb{R}_{d} \rightarrow \mathbb{T}$ is the continuous extension of $w \circ P$.

Proof. The claim is clearly true if $P=0$, so assume that $P$ has degree $n \geq 1$ and that the claim holds for all polynomials of smaller degree. Let $p$ be an idempotent in $\left(\beta \mathbb{R}_{d},+\right)$. For any $x$ and $y$ in $\mathbb{R}, P(y+x)=P(y)+Q(x, y)+P(x)$, where, for any fixed $y, Q(x, y)$ is a polynomial in $x$ with zero constant term and degree less than $n$. Taking limits as $x$ tends to $p$, we get that for all $y \in \mathbb{R}$,

$$
\widetilde{w \circ P}(y+p)=w(P(y))+\widetilde{w \circ P}(p) .
$$

Taking limits as $y$ tends to $p$, we get from $(*)$ that $\widetilde{w \circ P}(p)=0$ so that for all $y \in \mathbb{R}, \widetilde{w \circ P}(y+p)=w(P(y))$. Then taking limits as $y$ tends to $q$, we get $\widetilde{w \circ P}(q+p)=\widetilde{w \circ P}(q)$.

Notice that the following lemma does not assert that the set $A$ is nonempty. It does say that if it is nonempty, then it is big.

Lemma 3.6. Let $v \in \mathbb{N}$ and for $u \in\{1,2, \ldots, v\}$, let $P_{u}$ be a polynomial with real coefficients and zero constant term. Let $S$ be a subsemigroup of $\mathbb{R}_{d}$, let $U$ be an open subset of $\mathbb{T}^{v}$, and let $A=\left\{x \in S:\left(w\left(P_{1}(x)\right), w\left(P_{2}(x)\right), \ldots, w\left(P_{v}(x)\right)\right) \in U\right\}$. Then for all $x \in A,-x+A$ is an $I P^{*}$ set in $S$.

Proof. It suffices to prove the result in case $v=1$, so let $U$ be an open subset of $\mathbb{T}$, let $A=\left\{x \in S: w\left(P_{1}(x)\right) \in U\right\}$, let $x \in A$, and let $p$ be an idempotent in $\beta \mathbb{R}_{d}$. Let $f=w \circ P \circ \lambda_{x}$ and let $\tilde{f}: \beta \mathbb{R}_{d} \rightarrow \mathbb{T}$ be its continuous extension. Since $\tilde{f}$ is continuous, $\tilde{f}^{-1}[U]$ is a neighborhood of $p$ in $\beta \mathbb{R}_{d}$. So $-x+A=\{s \in S$ : $\left.w\left(P_{1}(x+s)\right) \in U\right\} \in p$.

\section{ACKNOWLEDGEMENT}

The authors want to thank the referee for a very helpful report which led to substantial improvements in the paper.

\section{REFERENCES}

1. J. Auslander, Minimal flows and their extensions, North Holland, Amsterdam, 1988. MR $956049(89 \mathrm{~m}: 54050)$

2. V. Bergelson, Ergodic Ramsey Theory - an update, London Math. Soc. Lecture Note Series 228, Cambridge University Press (1996), 1-61. MR1411215 (98g:28017)

3. V. Bergelson, Minimal idempotents and ergodic Ramsey Theory, London Math. Soc. Lecture Note Series 310, Cambridge University Press (2003), 8-39. MR2052273 (2006b:37022)

4. V. Bergelson, Ultrafilters, IP sets, dynamics, and combinatorial number theory, Contemporary Math. 530, Amer. Math. Soc. (2010), 23-47. MR2757532

5. V. Bergelson and N. Hindman, Nonmetrizable topological dynamics and Ramsey Theory, Trans. Amer. Math. Soc. 320 (1990), 293-320. MR.982232 (90k:03046)

6. V. Bergelson, N. Hindman, and R. McCutcheon, Notions of size and combinatorial properties of quotient sets in semigroups, Topology Proceedings 23 (1998), 23-60. MR 1743799 (2001a:20114)

7. V. Bergelson and A. Leibman, Distribution of values of bounded generalized polynomials, Acta Mathematica 198 (2007), 155-230. MR2318563(2008m:11149)

8. C. Chou, On a geometric property of the set of invariant means on a group, Proc. Amer. Math. Soc. 30 (1971), 296-302. MR0283584(44:815) 
9. T. Carlson, N. Hindman, J. McLeod, and D. Strauss, Almost disjoint large subsets of semigroups, Topology and its Applications 155 (2008), 433-444. MR2380928 (2008m:54050)

10. D. De, N. Hindman, and D. Strauss, A new and stronger Central Sets Theorem, Fund. Math. 199 (2008), 155-175. MR2410923 (2009c:05248)

11. R. Ellis, Distal transformation groups, Pacific J. Math. 8 (1958), 401-405. MR0101283(21:96)

12. H. Furstenberg, Recurrence in ergodic theory and combinatorical number theory, Princeton University Press, Princeton, NJ, 1981. MR603625 (82j:28010)

13. G. Hardy and J. Littlewood, Some problems of Diophantine approximation, Acta. Math. 37 (1914), 155-191. MR.1555098

14. N. Hindman, I. Leader, and D. Strauss, Infinite partition regular matrices - solutions in central sets, Trans. Amer. Math. Soc. 355 (2003), 1213-1235. MR 1938754 (2003h:05187)

15. N. Hindman and D. Strauss, Algebra in the Stone-Cech compactification: theory and applications, de Gruyter, Berlin, 1998. MR.1642231 (99j:54001)

16. L. Kronecker, Die Periodensysteme von Funktionen Reeller Variablen, Berliner Situngsberichte (1884), 1071-1080.

17. L. Kuipers and H. Niederreiter, Uniform distribution of sequences, Wiley, New York, 1974. MR 0419394 (54:7415)

18. R. McCutcheon, Elemental methods in ergodic Ramsey theory, Springer-Verlag, Berlin, 1999. MR.1738544(2001c:05141)

19. H. Shi and H. Yang, Nonmetrizable topological dynamical characterization of central sets, Fund. Math. 150 (1996), 1-9. MR.1387952 (97j:54045)

20. H. Weyl, Über die Gleichverteilung von Zahlen mod eins, Math. Ann. 77 (1916), 313-352. MR 1511862

Department of Mathematics, Ohio State University, Columbus, Ohio 43210

E-mail address: vitaly@math.ohio-state.edu

Department of Mathematics, Howard University, 2400 Sixth Street, NW, WashingTON, DC 20059

E-mail address: nhindman@aol.com

Department of Pure Mathematics, University of Leeds, Leeds LS2 9J2, United KingDOM

E-mail address: d.strauss@hull.ac.uk 